\title{
MATHEMATICAL MODELING OF THE CONTACT INTERACTION OF FUEL ELEMENTS USING THE MORTAR METHOD
}

\author{
P.S. ARONOV ${ }^{1,2 *}$, M.P. GALANIN ${ }^{1,2}$, AND A.S. RODIN ${ }^{1,2}$ \\ ${ }^{1}$ Keldysh Institute of Applied Mathematics (Russian Academy of Sciences). Moscow, Russia \\ ${ }^{2}$ Bauman Moscow State Technical University. Moscow, Russia \\ * Corresponding author. E-mail: aronovps@ mail.ru
}

\section{DOI: 10.20948/mathmontis-2020-48-5}

Summary. The article discusses the implementation of the algorithm for solving axisymmetric contact problems of the thermoelasticity theory using the mortar method. This algorithm is used for numerical simulation of the contact interaction of several bodies under thermomechanical loading. The ill-conditioned system of linear algebraic equations obtained as a result of finite element discretization is numerically solved using the modified symmetric successive overrelaxation method (MSSOR), generalized to the case of contact of several bodies. The results of the algorithm application are demonstrated on a problem simulating some processes in a fuel element with a different number of bodies. The effect of the contacting bodies number and mesh steps on the number of iterations necessary to achieve a given accuracy while solving the system of equations is investigated.

\section{INTRODUCTION}

Accounting the contact interaction of various functional equipment units allows to obtain a more accurate estimation of the construction stress-strain analysis. The most promising and often used technique of the contact interaction studying is numerical methods, the leading place among which is occupied by the finite element method.

It is often not possible to use matched meshes while modeling the contact of a large number of bodies. The numerical solution of such problems is carried out using various algorithms, among which we can distinguish the domain decomposition method [1,2], the penalty method $[3,4]$, various versions of the Lagrange multiplier method [5,6], in particular, the mortar method $[7,8]$, based on the construction of a sufficiently detailed auxiliary mesh for determining the Lagrange multipliers in the case when the meshes are unmatched. Algorithms designed for modelling the interaction of bodies in dynamic problems can also be noted [9].

This paper discusses a sufficiently general statement of the contact interaction problem of several bodies is given and an implementation of an algorithm for numerically solving contact problems using the mortar method is presented. The block system of linear algebraic equations with a saddle point obtained as a result of the problem discretization is ill-conditioned, and a modified symmetric successive over-relaxation method is used to solve it, generalized to the case of contact interaction of several bodies.

The article considers the problem modeling some processes in a fuel element with a different number of contacting bodies on unmatched meshes. The dependence of iterations number on the selected finite element mesh and the number of bodies is analyzed.

2010 Mathematics Subject Classification: 74M15, 74S05, 74B05.

Key words and Phrases: Contact Problem of the Elasticity Theory, Finite Element Method, Mortar-method, Succesive Over-Relaxation Method. 


\section{MATHEMATICAL FORMULATION OF THE PROBLEM}

Let a group of axisymmetric thermoelastic contacting bodies (Fig. 1) be located in threedimensional space $\mathbb{R}^{3}$, occupying a domain $G=\bigcup_{\alpha} G_{\alpha}(\alpha$ is a body number index $)$, bounded by a piecewise smooth border $\partial G=\bigcup_{\alpha} \partial G_{\alpha}$.

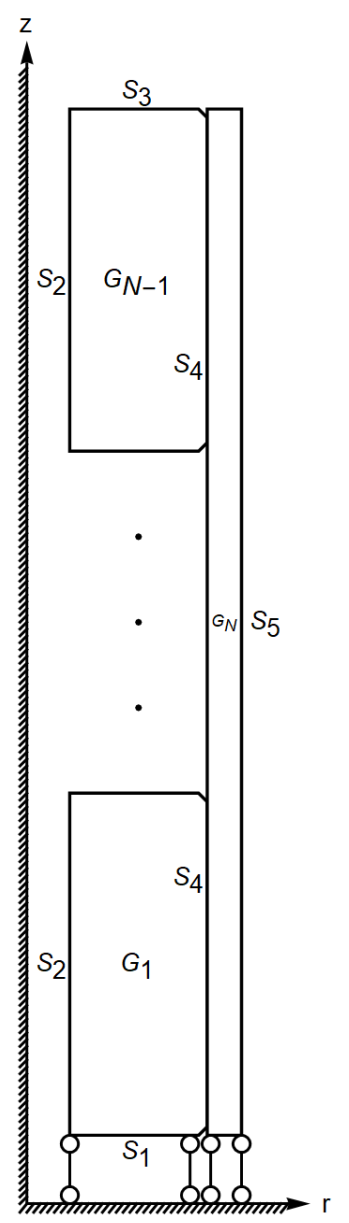

Figure 1. Scheme of contact interaction of several bodies

Consider the following problem: inside a cylindrical cladding $G_{N}$ there is a column of several placed on top of each other identical cylindrical pellets $G_{1}, \ldots, G_{N-1}$, having an inner hole and chamfers at both ends. Each pellet (except $G_{1}$ и $G_{N-1}$ ) comes into contact with two adjacent pellets and the cladding (it is believed that there is no initial gap between them). $S_{1}$ is the lower end of the lower pellet, $S_{2}$ is the inner surface of the pellets, $S_{3}$ is the top end of the top pellet, $S_{4}$ is the boundary between the inner surfaces of the pellets and the cladding, $S_{5}$ is the outer surface of the cladding. The lower ends of the cladding and lower pellets are fixed vertically. There is no friction on the contact surfaces. This problem simulates some thermomechanical processes taking place in a fuel element. 
Suppose that the coupling effect (the dependence of temperature on the deformation of the body) can be neglected; therefore, we will solve the heat conduction problem separately, and use the obtained temperature field to solve the contact problem of the thermoelasticity theory.

Consider the following initial-boundary value problem for the nonlinear heat equation:

$$
\begin{gathered}
c(T) \rho \frac{\partial T}{\partial t}=\left(k_{i j}(T) T_{, j}\right)_{, i}+q(\mathbf{x}), \quad \mathbf{x} \in G ; \\
T(\mathbf{x}, 0)=T_{0}(\mathbf{x}), \quad x \in G ; \\
-\left.n_{i} k_{i j}(T) T_{, j}\right|_{S_{1} \cup S_{2} \cup S_{3}}=q_{w}(\mathbf{x}, t), \quad \mathbf{x} \in S_{1} \cup S_{2} \cup S_{3}, \quad t>0 ; \\
-\left.n_{i} k_{i j}(T) T_{, j}\right|_{S_{4}}=\alpha(T)\left[T(\mathbf{x}, t)-T_{f}(\mathbf{x}, t)\right], \quad \mathbf{x} \in S_{4}, \quad t>0,
\end{gathered}
$$

where $c(T)$ is the specific heat capacity of the medium, $\rho$ is the medium density, $t$ is time, $k_{i j}$ is the thermal conductivity tensor components, $T_{, j}=\frac{\partial T}{\partial x_{j}}, q(\mathbf{x})$ is the power of internal sources (drains) of the body, $T_{0}(\mathbf{x})$ is the initial temperature, $T(\mathbf{x}, t)$ is temperature at time $t$, $n_{i}$ are the components of the unit vector of the external normal to the boundary $\partial G, q_{w}(\mathbf{x}, t)$ is the heat flux density on the surfaces $S_{1}, S_{2}, S_{3}, \alpha(T)$ is the heat transfer coefficient on the surface $S_{4}, T_{f}(\mathbf{x})$ is the temperature at a similar point lying on the opposite side of the contact pair.

The mathematical formulation of the contact problem of the elasticity theory for the case when there are no bulk forces includes the following relations [10] for each body $G_{\alpha} \subset \mathbb{R}^{3}$, participating in the contact $(i, j=\overline{1,3})$ :

- equilibrium equations

$$
\sigma_{j i, j}(\mathbf{u})=0, \quad \mathbf{x} \in G_{\alpha} ;
$$

- kinematic boundary conditions

$$
\mathbf{u}(\mathbf{x})=\mathbf{u}_{\mathbf{0}}(\mathbf{x}), \quad \mathbf{x} \in S_{1} ;
$$

- force boundary conditions

$$
\sigma_{j i}(\mathbf{u}) n_{j}=g_{i}(\mathbf{x}), \quad x \in S_{3} \cup S_{4} ;
$$

- Cauchy relations

$$
\varepsilon_{i j}(\mathbf{x})=\frac{1}{2}\left(u_{i, j}(\mathbf{x})+u_{j, i}(\mathbf{x})\right), \quad \mathbf{x} \in G_{\alpha} ;
$$

- governing equations (Hooke's law)

$$
\sigma_{i j}(\mathbf{x})=C_{i j k l}\left(\varepsilon_{k l}(\mathbf{x})-\varepsilon_{k l}^{0}(\mathbf{x})\right), \quad \mathbf{x} \in G_{\alpha} ;
$$

- kinematic contact condition 


$$
u_{n}^{\alpha_{1}}(\mathbf{x})=-u_{n}^{\alpha_{2}}(\mathbf{x}), \quad x \in S_{k}^{\alpha_{12}}
$$

- force contact condition

$$
\sigma_{n}^{\alpha_{1}}(\mathbf{x})=\sigma_{n}^{\alpha_{2}}(\mathbf{x}) \leqslant 0, \quad \mathbf{x} \in S_{k}^{\alpha_{12}},
$$

where $x_{i}$ are the coordinates of the vector $x \in G_{\alpha} ; \sigma_{i j}$ are the stress tensor components; $\varepsilon_{k l}$ are the strain tensor components; $\varepsilon_{k l}^{0}$ are the components of the initial strain tensor (for a thermoelastic body such are thermal strains); $u_{i}$ are the displacement vector components; $C_{i j k l}$ are the components of the elastic constants tensor; $g_{i}$ are the surface force vector components; $n_{j}$ are the components of the external normal vector to the corresponding surface $S_{j} ; u_{n}^{\alpha_{i}}$ are the projections of displacement vectors of boundary points on the direction of the external normal $n$ to the body $\alpha_{i}$ boundary; $\sigma_{n}^{\alpha_{i}}$ are the projections of stress vectors on the directions of external normals $n_{i}$.

The conditions of contact interaction with respect to displacements and stresses must be fulfilled when solving the problem on the contact surfaces of bodies.

For the case under consideration of the axisymmetric formulation of the problem, the vectors of stresses $\boldsymbol{\sigma}$, strains $\boldsymbol{\varepsilon}$, и and displacements $\mathbf{u}$ cylindrical coordinate system are written as follows:

$$
\boldsymbol{\sigma}=\left\{\begin{array}{c}
\sigma_{r} \\
\sigma_{z} \\
\sigma_{\theta} \\
\tau_{r \theta}
\end{array}\right\}, \quad \boldsymbol{\varepsilon}=\left\{\begin{array}{c}
\varepsilon_{r} \\
\varepsilon_{z} \\
\varepsilon_{\theta} \\
\gamma_{r \theta}
\end{array}\right\}, \quad \mathbf{u}=\left\{\begin{array}{l}
u_{r} \\
u_{z}
\end{array}\right\} .
$$

The solution of the problem (2)-(8) is equivalent to [11] minimizing the functional

$$
\Pi=\frac{1}{2} \int_{G} \boldsymbol{\sigma}^{T} \varepsilon d G-\int_{S} \mathbf{u}^{T} \mathbf{g} d S+\int_{S_{k}} \lambda_{n}\left(u_{n}^{2}(\mathbf{x})-u_{n}^{1}(\mathbf{x})\right) d S
$$

when fulfilling the kinematic boundary conditions (3), where $\lambda_{n}$ are the Lagrange multipliers, which are projections of the stress vectors on the directions of the external normals, $u_{n}=u_{r} n_{r}+u_{z} n_{z}$.

\section{BASIC MATRIX RELATIONS OF THE FINITE ELEMENT METHOD}

For the numerical solution of the problem (2)-(8) we will use the finite element method. The finite element mesh consists of second-order quadrangular elements.

The components $u_{r}^{(e)}, u_{z}^{(e)}$ of the displacement vector $\mathbf{u}$ inside the finite element with the index $e$ are determined using the dependence

$$
\left\{\begin{array}{c}
u_{r}^{(e)} \\
u_{z}^{(e)}
\end{array}\right\}=[N]^{(e)}\{u\}^{(e)}
$$


where $[N]^{(e)}$ is the matrix of the shape functions [12] of the finite element with the index $e$, and $\{u\}^{(e)}$ is the the combined vector of displacement components in all nodes of the finite element with the number $e$.

Relations between deformations and displacements are written as follows [12]:

$$
\{\varepsilon\}^{(e)}=[B]^{(e)}\{u\}^{e},
$$

where $[B]^{(e)}$ is the gradient matrix [11] of the finite element with the index $e$.

Stresses are expressed through deformations using the Hooke law:

$$
\{\sigma\}^{(e)}=\left[D_{\alpha}\right]^{(e)}\{\varepsilon\}^{(e)},
$$

or, taking into account (10),

$$
\{\sigma\}^{(e)}=\left[D_{\alpha}\right]^{(e)}[B]^{(e)}\{u\}^{(e)},
$$

where $\left[D_{\alpha}\right]^{(e)}$ is the local elasticity matrix of the finite element [12] with the index $e$ for the body with the index $\alpha$. For the axisymmetric formulation of the problem the matrix $\left[D_{\alpha}\right]^{(e)}$ is written as follows:

$$
\left[D_{\alpha}\right]^{(e)}=\frac{E}{(1+v)(1-2 v)}\left(\begin{array}{cccc}
1-v & v & v & 0 \\
v & 1-v & v & 0 \\
v & v & 1-v & 0 \\
0 & 0 & 0 & \frac{1-2 v}{2}
\end{array}\right),
$$

where $E$ is the Young's modulus and $v$ is the Poisson's ratio.

\section{APPLICATION OF THE MORTAR METHOD FOR SOLVING CONTACT PROBLEMS}

The mortar method for solving contact problems of the elasticity theory is based on the independent finite element discretization of disjoint subdomains. The meshes on these subdomains are, generally speaking, unmatched on the contact line, and the continuity of the solution is achieved through the use of Lagrange multipliers [13]. Among the main advantages of the mortar method, the possibility of independent selection of various types of finite elements and shape functions both at the boundaries of contacting bodies and during integration along the contact line can be noted.

To simplify the recording, we restrict ourselves to the case of two bodies with one pair of contact surfaces. Let the body $G_{m}$ be master and the body $G_{s}$ be slave. The contact line from the side of the body $G_{m}$ is denoted by $\Gamma_{m}$ and from the side of the body $G_{s}$ is denoted by $\Gamma_{s}$. We consider one-dimensional second order finite elements on the contact lines $\Gamma_{m}$ and $\Gamma_{s}$. From the nodes of these elements on the contact line $\Gamma_{m}$ we draw normals to contact line $\Gamma_{s}$. 
For the finite elements formed at the intersection of the normals and $\Gamma_{s}$ we will carry out further integration, considering them also as one-dimensional quadratic elements with similar shape functions. The division of bodies into master/slave is largely conditional and non-obvious, but ultimately this choice determines the discretization of Lagrange multipliers [14].

Consider the following integral:

$$
\int_{\Gamma} \lambda^{T}\left(\mathbf{u}_{\mathbf{m}}-\mathbf{u}_{\mathbf{s}}\right) d \gamma=\sum_{i=1}^{k_{m}} \int_{\Gamma_{m i}} \lambda^{T} \mathbf{u}_{\mathbf{m}} d \gamma-\sum_{i=1}^{k_{s}} \int_{\Gamma_{s i}} \lambda^{T} \mathbf{u}_{s} d \gamma,
$$

where $\Gamma=\Gamma_{m} \cup \Gamma_{s}, k_{m}$ and $k_{s}$ are the total number of finite elements into which the contact lines $\Gamma_{m}$ and $\Gamma_{s}$ are divided, respectively, the vectors $\mathbf{u}_{\mathbf{m}}$ and $\mathbf{u}_{\mathbf{s}}$ consist of the normal components of the displacement vectors of the finite element nodes on the contact lines $\Gamma_{m}$ and $\Gamma_{s}$, the vector $\lambda$ consists of Lagrange multipliers corresponding to the projections of stress vectors on the directions of external normals on the contact line $\Gamma_{s}$. Inside the finite element with the index $(e)$ values of $\lambda_{n}, u_{s}$ and $u_{m}$ are expressed as follows:

$$
\lambda_{n}=\left[N_{\lambda}\right]^{(e)}\{\lambda\}^{(e)}, \quad u_{s}=\left[N_{s}\right]^{(e)}\left\{u_{s}\right\}^{(e)}, \quad u_{m}=\left[N_{m}\right]^{(e)}\left\{u_{m}\right\}^{(e)},
$$

where $\left[N_{\lambda}\right]^{(e)},\left[N_{s}\right]^{(e)},\left[N_{m}\right]^{(e)}$ are the matrices of the shape functions of the one-dimensional quadratic element with the index $(e)$.

Minimization of functional (9) together with integral (11) leads to the formation of the following system of linear algebraic equations [15]:

$$
\left(\begin{array}{cccccc}
A_{11} & \mathbf{0} & \mathbf{0} & \ldots & \mathbf{0} & M_{1} \\
\mathbf{0} & A_{22} & \mathbf{0} & \ldots & \mathbf{0} & M_{2} \\
\ldots & \ldots & \ldots & \ldots & \ldots & \ldots \\
\mathbf{0} & \mathbf{0} & \ldots & A_{(N-1)(N-1)} & \mathbf{0} & M_{N-1} \\
\mathbf{0} & \mathbf{0} & \ldots & \mathbf{0} & A_{N N} & M_{N} \\
M_{1}^{T} & M_{2}^{T} & \ldots & M_{N-1}^{T} & M_{N}^{T} & \mathbf{0}
\end{array}\right)\left\{\begin{array}{c}
\mathbf{u}_{1} \\
\mathbf{u}_{\mathbf{2}} \\
\vdots \\
\mathbf{u}_{\mathrm{N}-\mathbf{1}} \\
\mathbf{u}_{\mathbf{N}} \\
\boldsymbol{\lambda}
\end{array}\right\}=\left\{\begin{array}{c}
\mathbf{R}_{\mathbf{1}} \\
\mathbf{R}_{\mathbf{2}} \\
\vdots \\
\mathbf{R}_{\mathbf{N}-\mathbf{1}} \\
\mathbf{R}_{\mathbf{N}} \\
\mathbf{0}
\end{array}\right\},
$$

where

$$
\begin{aligned}
& {\left[A_{i i}\right]=\sum_{e=1}^{k_{i}}\left[a_{G}\right]^{(e) T}\left(\int_{G_{i}}[B]^{(e) T}\left[D_{i}\right]^{(e)}[B]^{(e)} d G\right)\left[a_{G}\right]^{(e)},} \\
& \left\{R_{i}\right\}=\sum_{e=1}^{k_{i}}\left[a_{S}\right]^{(e) T}\left([N]^{(e) T}\left[g_{i}\right]^{(e)} d V\right) \\
& \lambda=\left(\begin{array}{lllll}
\lambda_{1} & \lambda_{2} & \ldots & \lambda_{q-1} & \lambda_{q}
\end{array}\right)^{T}, \\
& M_{i}=\left(\begin{array}{lllllllllllllll}
0 & \ldots & 0 & M_{i 1} & 0 & \ldots & 0 & M_{i j} & 0 & \ldots & 0 & M_{i p} & 0 & \ldots & 0
\end{array}\right),
\end{aligned}
$$

where $N$ is the number of bodies, $q$ is the number of contact pairs and in the columns of the 
matrices $M_{i}$ corresponding to the numbers of the bodies in contact with the body with the number $i$, are the following matrices

$$
\left[M_{i j}\right]=\sum_{e=1}^{k_{i}}\left[a_{S}\right]^{(e) T}\left(\int_{\Gamma_{s i}}[N]^{(e) T}[N]^{(e)} d \gamma\right)\left[a_{S}\right]^{(e)} .
$$

Here $\left[a_{G}\right]^{(e)},\left[a_{S}\right]^{(e)}$ are the matrices of geometric interactions of the finite element with the index $(e),\left[D_{i}\right]^{(e)}$ are the local elasticity matrices of the finite element with the index $(e),\left\{g_{i}\right\}$ are the local surface force vectors, $k_{i}$ are the number of finite elements into which the body $G_{i}$ is divided.

Matrices $A_{i i}$ have the dimension $n_{i} \times n_{i}, i=1, \ldots, N, n_{i}$ is the the number of unknown displacements in the $i$-th body, $n=n_{1}+\ldots+n_{N}$ is the total number of unknown displacements, matrices $M_{i}$ have the dimension $n_{i} \times m, m=m_{1}+\ldots+m_{q}$ is the total unknown Lagrange multipliers, matrices $M_{i j}$ have the dimension $n_{i} \times m_{j}, m_{j}$ is the the number of unknown Lagrange multipliers for the $j$-th contact pair, which includes the $i$-th body.

\section{ALGORITHM FOR SOLVING THE SYSTEM OF LINEAR ALGEBRAIC EQUATIONS}

The system of linear algebraic equations (12) with a saddle point is ill-conditioned and has a zero block on the main diagonal, therefore, for its numerical solution we will use modified symmetric successive over-relaxation method (MSSOR) [16,17], generalized to the case of contact of several bodies:

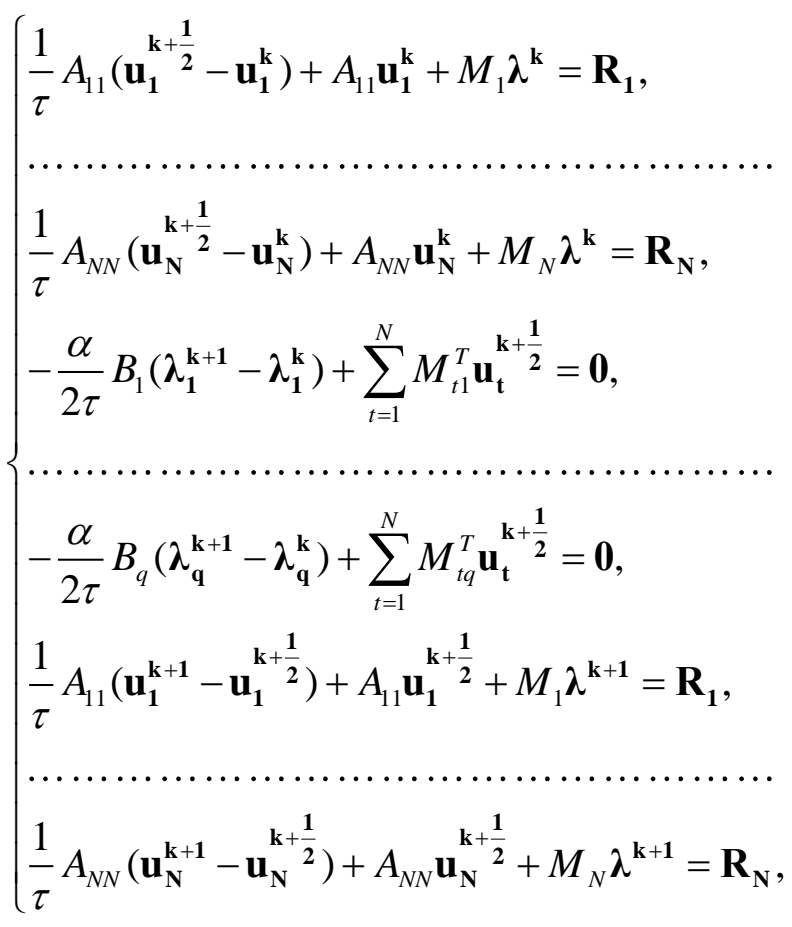


where $k$ is an iteration number, $\alpha$ and $\tau$ are the iterative parameters.

Before the first iteration, it is necessary to set the initial (zero) value to the vector of Lagrange multipliers $\lambda$ and then calculate the displacement vectors $\mathbf{u}_{\mathbf{i}}$ from the first $m$ equations of system (12).

Using the scheme (13) allows us to reduce the solution of the general ill-conditioned system of equations for all contacting bodies to the sequential solution of three blocks of systems of equations: $N$ systems for calculating $\mathbf{u}_{\mathbf{i}}^{\mathbf{k}+\frac{1}{2}}, q$ systems for calculating $\lambda_{\mathbf{i}}^{\mathbf{k}+1}$ и $N$ systems for calculating $\mathbf{u}_{\mathbf{i}}^{\mathbf{k}+1}$. Within each of these blocks, systems of equations can be solved independently of each other, including in parallel. All these systems of equations are solved using the conjugate gradient method. Matrices selected as preconditioners are $B_{i}=\sum_{j=1}^{N} M_{j i}^{T}\left(\operatorname{diag}\left\{A_{j j}\right\}\right)^{-1} M_{j i}$, and the values of the iterative parameters are set as follows: $\alpha=0,05, \tau=0,5$.

\section{RESULTS OF THE NUMERICAL SOLUTION}

First, we will solve the initial-boundary value problem of the heat equation (1), and we will use the obtained temperature field to solve the contact problem of the elasticity theory (2)-(8). In the pellets, a constant heat release is set, and the temperature in all nodes of the cladding is assumed to be constant $(623 \mathrm{~K})$. Constant pressure $p_{1}=10 \mathrm{MPa}$ is set on the outer surface of the cladding, and constant pressure $p_{2}=50 \mathrm{MPa}$ is set on the upper surface of the upper pellet. The pellets are made of uranium dioxide, the cladding is made of an alloy of zirconium. The elastic moduli, thermal expansion coefficients, specific heat capacity and thermal conductivity of both materials are temperature dependent, and the Poisson's ratios and density are constant.

We will carry out a series of calculations with a different number of pellets. For the case of five pellets, we present the distribution of radial and axial displacements and stresses at the contact boundary between the pellets and the cladding. We will consider unmatched meshes: the pellets $G_{1}, \ldots, G_{N-1}$ are divided into 40 elements in the $r$ direction and into 80 elements in the $z$ direction, and the cladding $G_{N}$ is divided into 10 elements in the $r$ direction and into 400 elements in the $z$ direction.

The considered problem has the following specific features:

- at the contact boundaries between the pellets, almost all finite elements (except for a few elements near the inner surface) exit the contact;

- axial displacements reach significant values: for example, for the case of 100 pellets, the upper pellet displacements relative to its initial position by an amount comparable to the size of several pellets.

Fig. 2-3 show the graphs of the distributions of radial and axial displacements at the boundary between the pellets and the cladding. Fig. 4-5 show the graphs of the distributions of radial and axial stresses at the boundary between the pellets and the cladding. The graphs of radial displacements stresses are visually indistinguishable and coincide everywhere, except for the vicinity of the chamfers of the pellets; no oscillations are observed. 


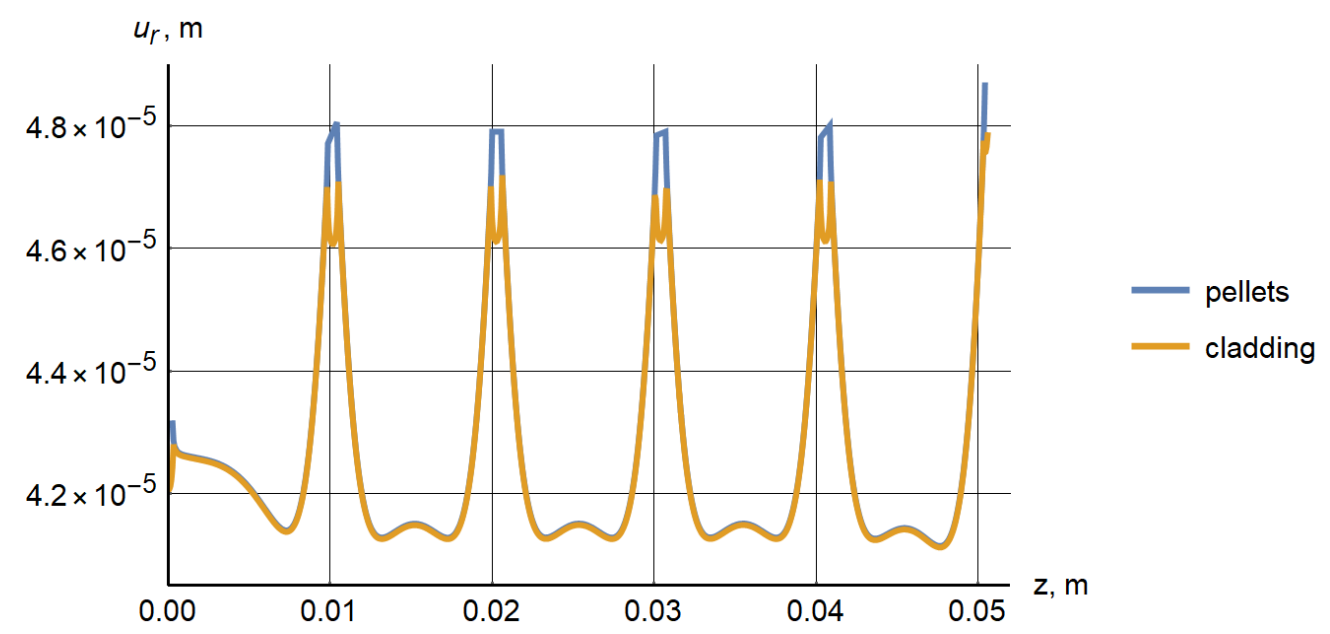

Figure 2. Radial displacements $u_{r}(z)$ in the elements nodes

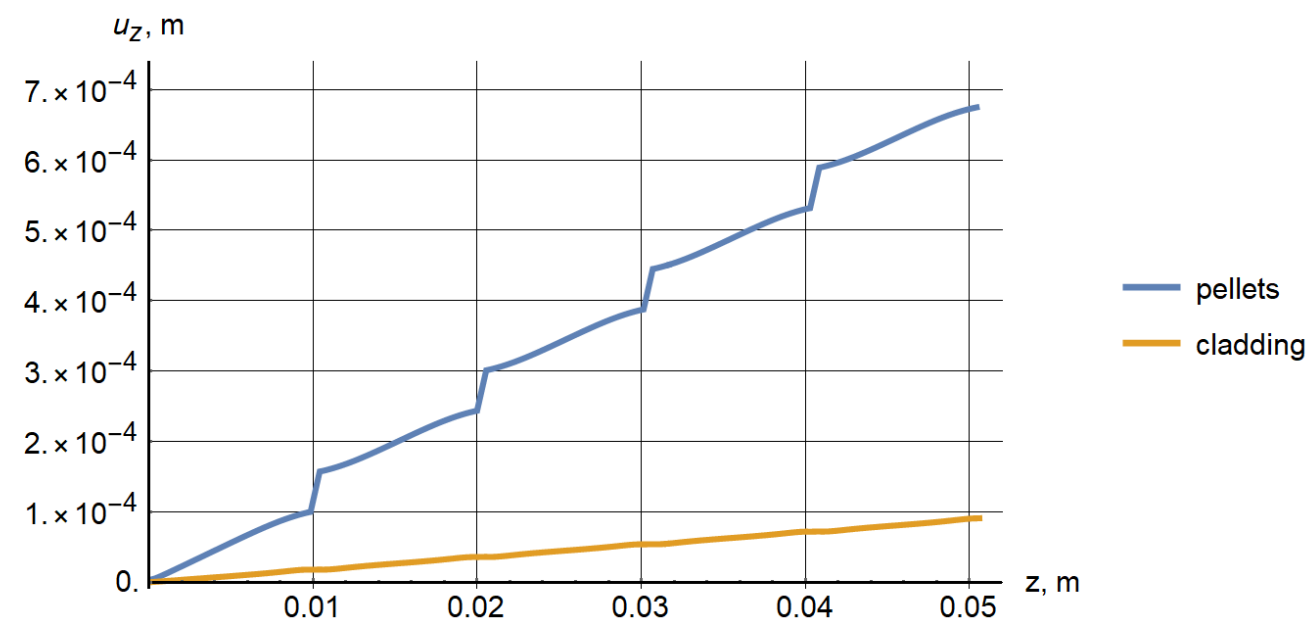

Figure 3. Axial displacements $u_{z}(z)$ in the elements nodes

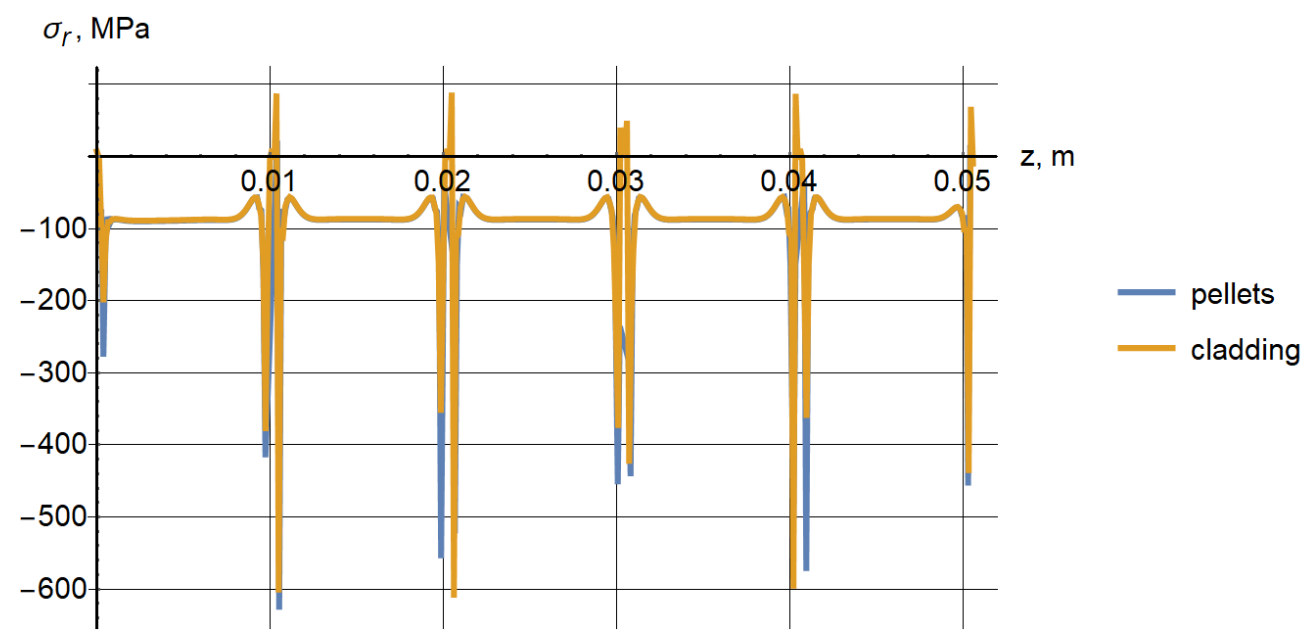

Figure 4. Radial stresses $\sigma_{r}(z)$ in the elements nodes 


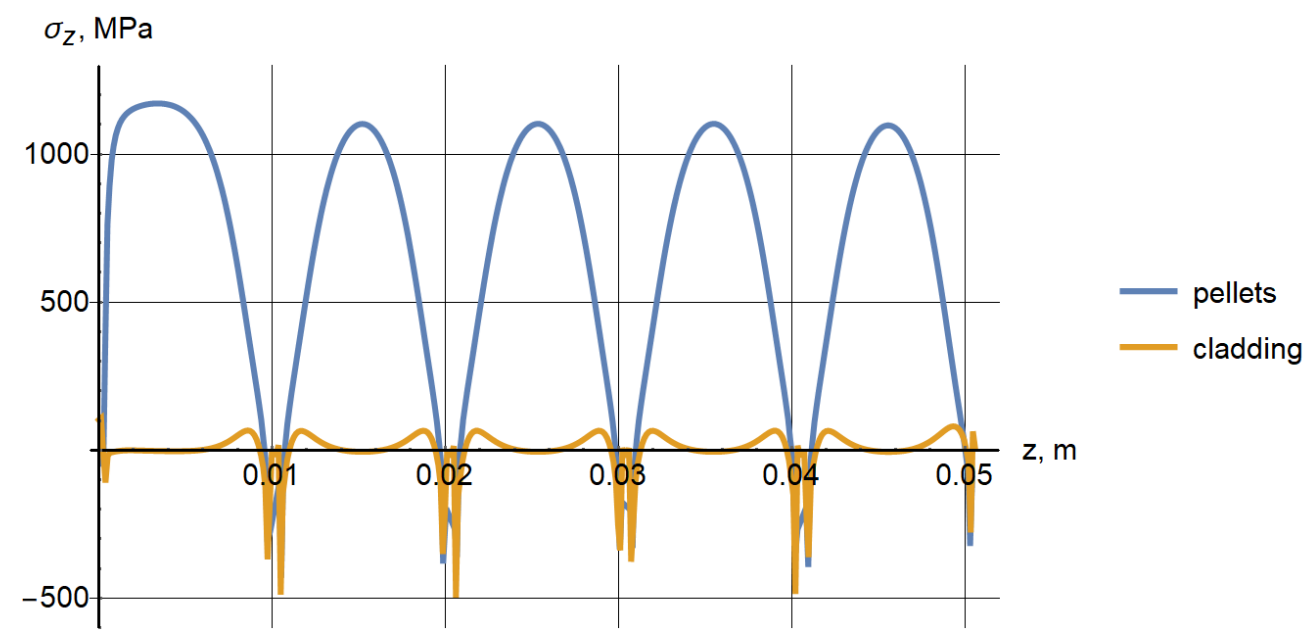

Figure 5. Axial stresses $\sigma_{z}(z)$ in the elements nodes

For the case of 100 pellets, we present fragments of two-dimensional distributions of displacements and stresses in the middle of a pillar column.

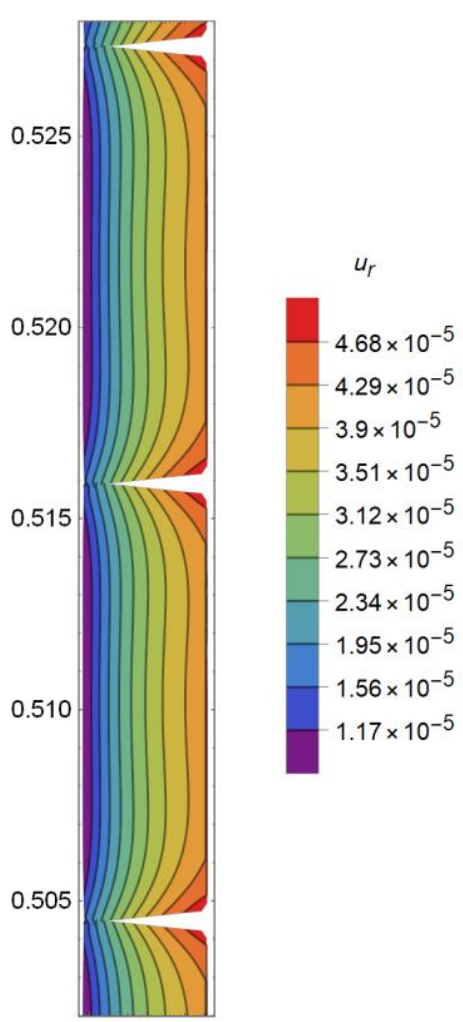

a)

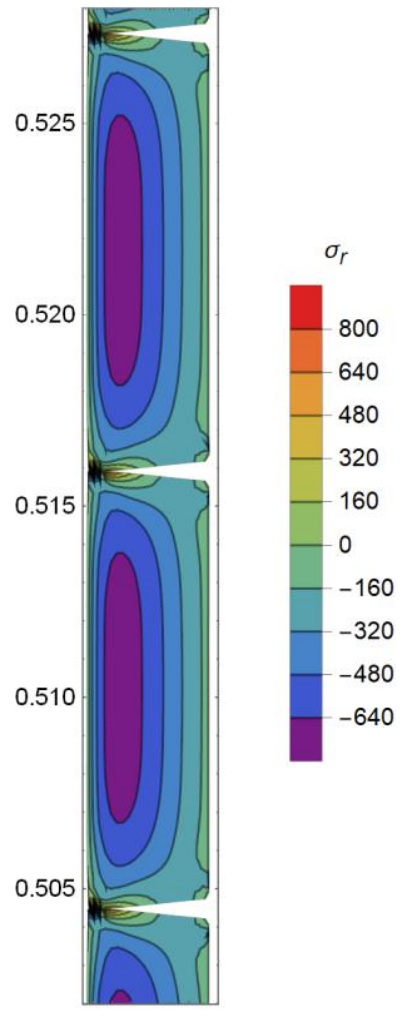

b)

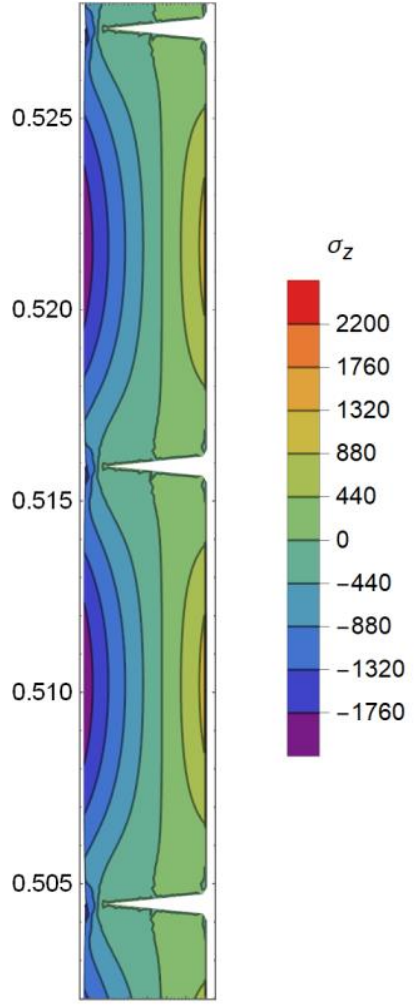

c)

Figure 6. Two-dimensional distributions in the elements nodes of the 49th and 50th pellets: a) radial displacements, b) — radial stresses, c) — axial stresses 


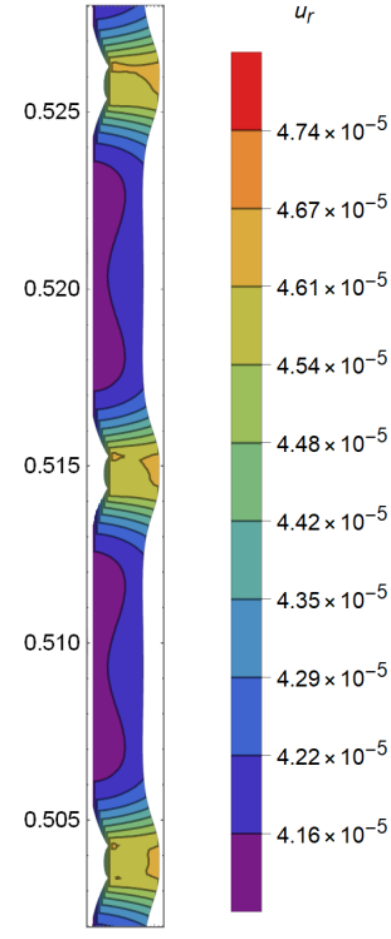

a)

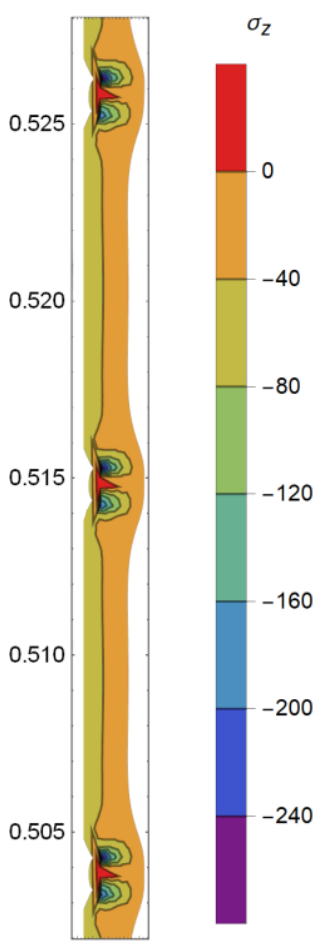

b)

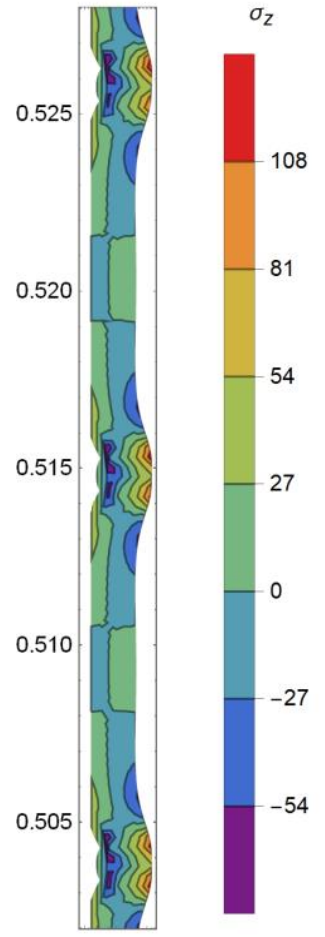

c)

Figure 7. Two-dimensional distributions in the elements nodes of the cladding: a) — radial displacements, b) — radial stresses, c) — axial stresses

Fig. 6-7a show two-dimensional distributions of radial displacements, and Fig. 6-7b and 6-7c show two-dimensional distributions of radial and axial stresses for the case of 100 pellets. Fragments of the distributions corresponding to the 49th and 50th pellets are shown, and when constructing deformed bodies, the applied displacements were increased by 10 times for pellets and 50 times for the cladding for clarity.

We will carry out a series of calculations with three different unmatched meshes:

- Mesh 1: the pellets are divided into 20 elements in the $r$ direction and into 40 elements in the $z$ direction, the cladding is divided into 5 elements in the $r$ direction,

- Mesh 2: the pellets are divided into 40 elements in the $r$ direction and into 80 elements in the $z$ direction, the cladding is divided into 10 elements in the $r$ direction,

- Mesh 3: the pellets are divided into 80 elements in the $r$ direction and into 160 elements in the $z$ direction, the cladding is divided into 20 elements in the $r$ direction.

In the $z$ direction the cladding is divided into the required number of elements in proportion to the number of pellets. For example, for five pellets, this will be 200, 400, and 800 elements for the three variants of the mesh. Fig. 8-9 show the graphs of the distribution of axial stress at the contact boundary between the 3 rd pellet and the cladding. It is seen that when using a coarser mesh, oscillations arise near the chamfers, with an increase in the number of elements, the amplitude of the oscillations decreases and on the smallest mesh they disappear. 


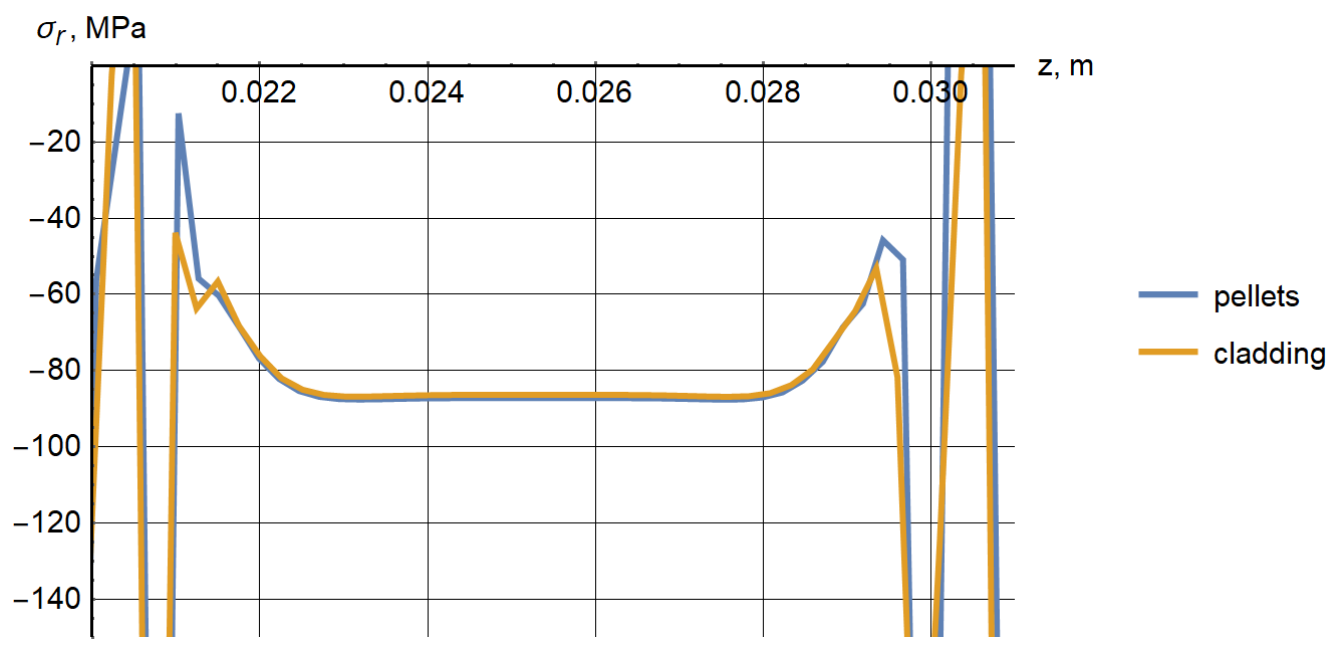

Figure 8. Radial stresses $\sigma_{r}(z)$ in the elements nodes of the 3rd pellet (mesh 1)

$$
\sigma_{r}, \mathrm{MPa}
$$
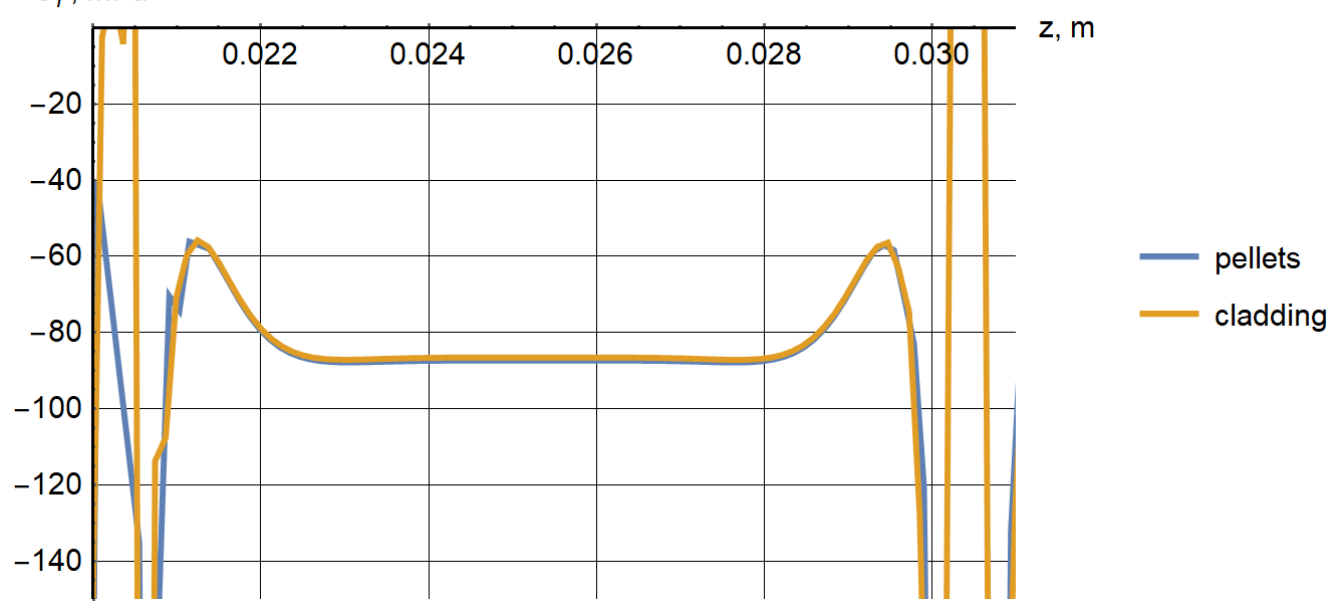

Figure 9. Radial stresses $\sigma_{r}(z)$ in the elements nodes of the 3rd pellet (mesh 2) $\sigma_{r}, \mathrm{MPa}$

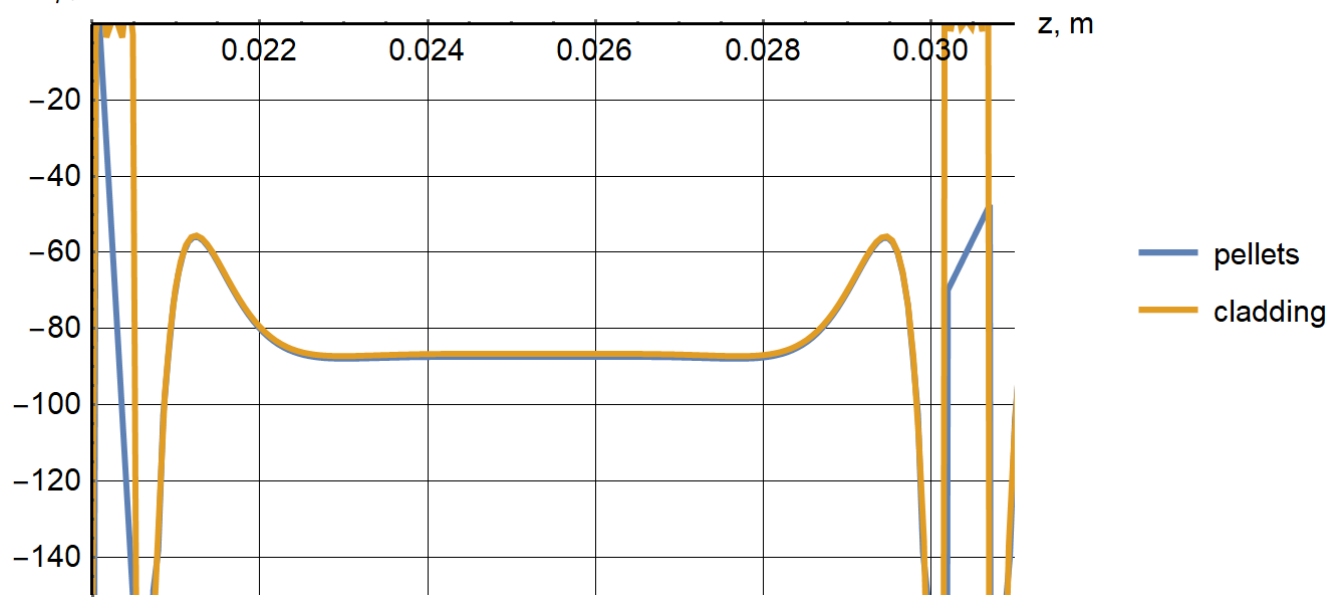

Figure 10. Radial stresses $\sigma_{r}(z)$ in the elements nodes of the 3rd pellet (mesh 3) 
Let us compare the number of iterations necessary to achieve the given accuracy of solving the system of equations (12), with a different number of pellets. In this case, the relative accuracy is calculated as follows:

$$
\varepsilon=\sqrt{\frac{\sum_{i} S_{i} \frac{\left(\hat{u}_{r_{i}}-u_{r_{i}}\right)^{2}+\left(\hat{u}_{z_{i}}-u_{z_{i}}\right)^{2}}{u_{r_{i}}^{2}+u_{z_{i}}^{2}}}{\sum_{i} S_{i}}}
$$

where $\hat{u}_{r_{i}}, \hat{u}_{z_{i}}$ are the values of radial and axial displacements at a new iteration, $S_{i}$ is the sum of the areas of the finite elements, which includes the considered node, divided by the number of nodes in the finite element.

\begin{tabular}{|c|c|c|c|}
\hline$\varepsilon$ & Mesh 1 & Mesh 2 & Mesh 3 \\
\hline $10^{-2}$ & 5 & 5 & 6 \\
\hline $10^{-3}$ & 6 & 7 & 8 \\
\hline $10^{-4}$ & 12 & 15 & 21 \\
\hline $10^{-5}$ & 29 & 38 & 56 \\
\hline
\end{tabular}

Table 1 . The number of iterations required to achieve accuracy of $\varepsilon$ (5 pellets)

\begin{tabular}{|c|c|c|c|}
\hline$\varepsilon$ & Mesh 1 & Mesh 2 & Mesh 3 \\
\hline $10^{-2}$ & 8 & 10 & 12 \\
\hline $10^{-3}$ & 14 & 18 & 22 \\
\hline $10^{-4}$ & 30 & 39 & 52 \\
\hline $10^{-5}$ & 64 & 80 & 94 \\
\hline
\end{tabular}

Table 2. The number of iterations required to achieve accuracy of $\varepsilon$ (25 pellets)

\begin{tabular}{|c|c|c|c|}
\hline$\varepsilon$ & Mesh 1 & Mesh 2 & Mesh 3 \\
\hline $10^{-2}$ & 16 & 19 & 24 \\
\hline $10^{-3}$ & 25 & 31 & 40 \\
\hline $10^{-4}$ & 51 & 66 & 90 \\
\hline $5 \cdot 10^{-5}$ & 85 & 102 & 148 \\
\hline
\end{tabular}

Table 3. The number of iterations required to achieve accuracy of $\varepsilon$ (100 pellets)

Tables 1-3 show that with increasing accuracy, the number of bodies and decreasing mesh step, the number of iterations increases. For 100 pellets, maximum accuracy of $\varepsilon=5 \cdot 10^{-5}$ is achieved, there is no convergence for the accuracy of $\varepsilon=10^{-5}$.

Let us determine some empirical patterns of growth in the number of iterations depending on the number of pellets and nodes of the finite element model. With an increase in the number of nodes, the number of iterations increases in proportion to $n^{y}$, where $y \in(0,225 ; 0.275)$. The above range was obtained for calculations with a different number of bodies $(5,10,25,50,100$ pellets). It is known that when using the conjugate gradient method without preconditioning for 
matrices obtained by discretizing the Laplace operator, the number of iterations increases in proportion to $\sqrt{n}$ [18]. If unit matrices are used as preconditioners $B_{i}$, then there is no convergence. The selected preconditioner allows faster convergence compared to the conjugate gradient method for conventional matrices.

As the number of pellets increases, the number of iterations increases in proportion to $N^{x}$, where $x \in(0,37 ; 0,41)$. This range was obtained for calculations with three different meshes (Fig. 11). The number of iterations for the calculation with five pellets is somewhat inconsistent with the proposed regularity, therefore, $N=10$ was taken as a starting point for constructing the dependence graph of $C^{0,4}$. In real fuel elements, the number of pellets reaches several hundred; therefore, the proposed algorithm allows us to obtain a numerical solution with sufficient accuracy for a moderate number of iterations.

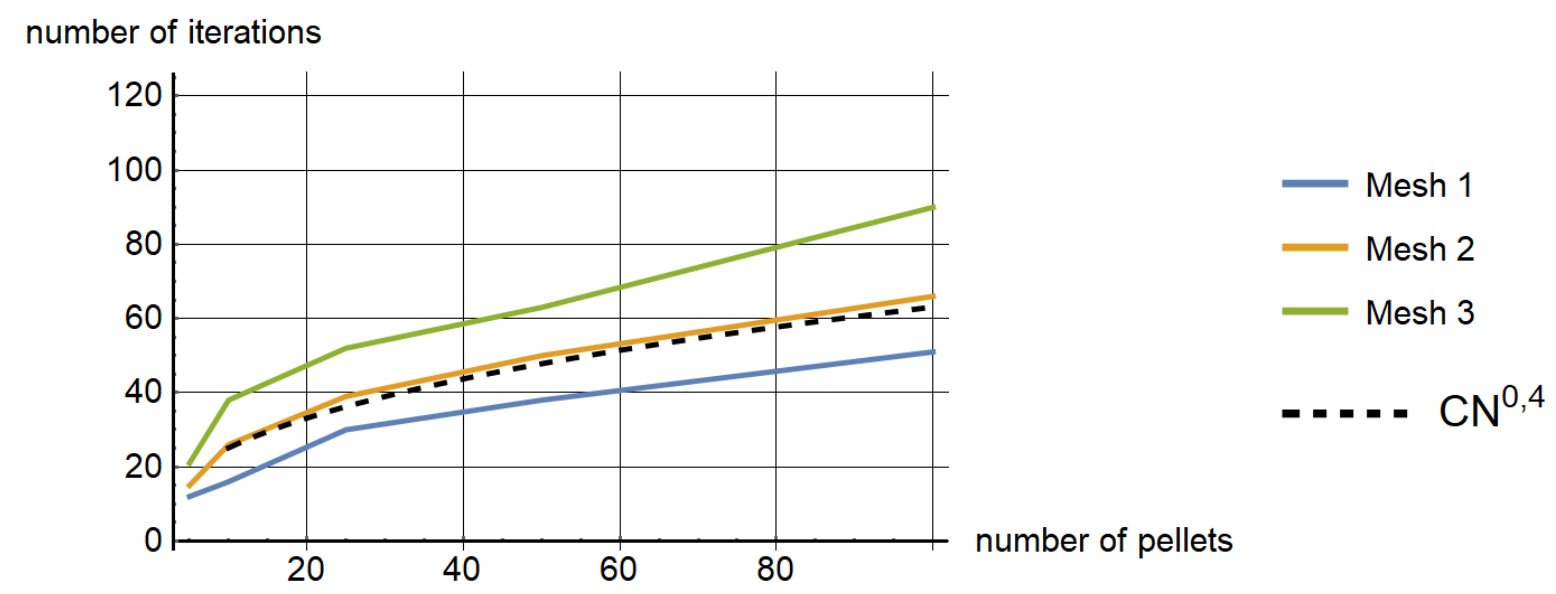

Figure 11. Dependence of the number of iterations on the number of pellets for $\varepsilon=10^{-4}$

\section{CONCLUSIONS}

The statement of the problem of contact interaction of a system of axisymmetric thermoelastic bodies under thermomechanical loading is presented. The results of the numerical implementation of the algorithm for solving the problem using the mortar method are presented on the example of a demonstration task that simulates some processes in a fuel element. A generalization of the algorithm for solving a system of linear algebraic equations arising as a result of discretization of the problem by the finite element method, in the case of contact of several bodies. A comparison is made of the number of iterations required to achieve a given accuracy of solving the system of equations, depending on the number of pellets and mesh steps. It is shown that the proposed method for the numerical solution of an ill-conditioned system of equations allows convergence to be achieved with sufficient accuracy for a moderate number of iterations. It is also demonstrated that with an increase in the number of elements, the amplitude of the axial stress oscillations arising near the chamfers of the pellets decreases.

Acknowledgements: The authors with deep gratitude and appreciation remember untimely departed D.Sc. in Engineering Stankevich Igor Vasilyevich. 


\section{REFERENCES}

[1] M.P. Galanin, A.V. Krupkin, V.I. Kuzneczov, V.V. Lukin, V.V. Novikov, A.S. Rodin, I.V. Stankevich, "Matematicheskoe modelirovanie termouprugoplasticheskogo kontaktnogo vzaimodejstviya sistemy tel", Mathematica Montisnigri, 30, 99-114 (2014).

[2] I.V. Stankevich, M.E. Yakovlev, Si Tu Xtet, "Razrabotka algoritma kontaktnogo vzaimodejstviya na osnove alterniruyushhego metoda Shvarcza", Vestnik MGTU im. N.E. Baumana. Ser. Estestvennye nauki. Specz. vyp. Prikladnaya matematika, 134-141 (2011).

[3] I. Babuska, "The finite element method with penalty", Mathematics of Computation, 27, 221-228 (1973).

[4] M.V. Mikhaylyuk, E.V. Strashnov, "Simulation of articulated rigid bodies with penalty method", Mathematica Montisnigri, 27, 91-106 (2013).

[5] P. Le Tallec, T. Sassi, "Domain decomposition with nonmatching grids: augmented Lagrangian approach", Mathematics of Computation, 64, 1367-1396 (1995).

[6] M.P. Galanin, P.V. Gliznucina, V.V. Lukin, A.S. Rodin, Varianty realizacii metoda mnozhitelej Lagranzha dlya resheniya dvumernyx kontaktnyx zadach, Preprint IPM, No. 89, (Moscow: KIAM), (2015). URL: http://library.keldysh.ru/preprint.asp?id=2015-89.

[7] P. Wriggers, Computational Contact Mechanics, Speinger-Verlag (2006).

[8] B.P. Lamichhane, Higher Order Mortar Finite Elements with Dual Largange Multiplier Spaces and Applications, Universitat Stuttgart (2006).

[9] E.I. Kraus, I.I. Shabalin, "The tool for high-velocity interaction and damage of solids", Mathematica Montisnigri, 39, 18-29 (2017).

[10] V.S. Zarubin, G.N. Kuvyrkin, Matematicheskie modeli mexaniki i elektrodinamiki sploshnoj sredy, Izd-vo MGTU im. N.E. Baumana (2008).

[11] L.A. Rozin, Variacionnye postanovki zadach dlya uprugix sistem, Izd-vo Leningradskogo un-ta (1978).

[12] O. Zenkevich, K. Morgan, Konechnye elementy i approksimaciya. Mir (1986).

[13] B.I. Wohlmuth, "A mortar finite element method using dual spaces for the Lagrange multiplier", SIAM Journal on Numerical Analysis, 38 (3), 989-1012 (2000).

[14] P.S. Aronov, A.S. Rodin, Matematicheskoe modelirovanie kontaktnogo vzaimodejstviya dvux uprugix tel s krivolinejnymi graniczami na nesoglasovannyx setkax, Preprint IPM, No. 87, (Moscow: KIAM), (2019). doi:10.20948/prepr-2019-87.

[15] I.V. Stankevich, P.S. Aronov, "Matematicheskoe modelirovanie kontaktnogo vzaimodejstviya dvux uprugix tel s pomoshhyu mortar-metoda", Matematika i matematicheskoe modelirovanie, 3, 26-44 (2018).

[16] Yu.V. Bychenkov, E.V. Chizhonkov, Iteracionnye metody resheniya sedlovyx zadach, BINOM (2010).

[17] P.S. Aronov, M.P. Galanin, A.S. Rodin, I.V. Stankevich, "Reshenie zadachi kontakta dvux uprugix tel mortar-metodom i metodom Shvarcza na nesoglasovannyx setkax", Tavricheskij vestnik informatiki i matematiki, 1, 24-42, (2019).

[18] Dzh. Demmel, Vychislitelnaya linejnaya algebra. Teoriya i prilozheniya, Mir (2001).

Received May 10, 2020 